Communications in Physics, Vol. 31, No. 3 (2021), pp. 259-268

DOI:10.15625/0868-3166/15821

\title{
ECONOMIZING NONLOCAL RESOURCE IN TWO-WAY REMOTE PREPARATIONS OF INEQUIVALENT QUANTUM STATES UNDER A COMMON CONTROL
}

\author{
NGUYEN BA AN ${ }^{1,2, \dagger}$
}

${ }^{1}$ Thang Long Institute of Mathematics and Applied Sciences,

Thang Long University, Nghiem Xuan Yem, Hoang Mai, Hanoi

${ }^{2}$ Center for Theoretical Physics, Institute of Physics, Vietnam Academy of Science and Technology, 18 Hoang Quoc Viet, Hanoi

E-mail: ${ }^{\dagger}$ annb@ $@$ thanglong.edu.vn

Received 6 January 2021

Accepted for publication 14 March 2021

Published 18 April 2021

\begin{abstract}
A recently proposed protocol for two parties to securely and simultaneously exchange inequivalent quantum states under the same control of a third party [Int. J. Theor. Phys. 60 (2021) 47] is revisited. It is shown in this paper that the required tasks are equally well accomplished with a nonlocal resource which is greatly economized by rationally exploiting local resources. Furthermore, the economized nonlocal resource with appropriate local operations on local resources also allows exchanging inequivalent states other than those previously considered.
\end{abstract}

Keywords: multiqubit entanglement; two-way remote state preparation; common control; GHZtype state; W-type state.

Classification numbers: 03.67.Hk; 03.65.Ud; 03.67.-a; 03.67.Bg.

\section{INTRODUCTION}

Entanglement $[1,2]$, thanks to its instantaneous nonclassical correlation and distanceindependent spooky action, has been recognized as an incredible nonlocal resource enabling, only by local operations and classical communication (LOCC), execution of various global tasks in the quantum world that have no counterparts in the classical world. In a global task within a quantum network there are a number of remote parties who are authorized to participate in the task. However, there are also unauthorized parties who aim to intervene into the task. Good quantum protocols for a given task should be designed such that only the authorized parties are able to (C)2021 Vietnam Academy of Science and Technology 
manage the task and any interventions of any unauthorized parties are detectable by the authorized parties. That is, a good protocol has to be secure with respect to any outsiders' attacks. Because only LOCC is allowed, entanglement must be shared beforehand among all the authorized parties, who are far apart from each other, and entanglement is treated as a nonlocal resource. For a perfect performance it commonly demands that the nonlocal resource is a maximally entangled state. More concretely, after having firmly shared a maximally entangled state, the authorized parties do not need to gather together but just stay in their own labs to do in-spot necessary operations (applying unitary operators or/and carrying out quantum measurements) and then publicly broadcast via insecure yet reliable classical communication channels the obtained measurements' outcomes followed by some conditioned local operations. As an illustration, for Alice to securely transfer to her remote partner Bob an unknown quantum information hidden in a quantum bit (qubit) without physically sending the qubit, the two parties have to share in advance a two-qubit maximally entangled state (called EPR pair which was used by Einstein, Podolsky and Rosen in their 1935's paper [1]). In this case the entanglement sharing implies that each party holds a qubit of the EPR pair. Then Alice makes a joint measurement (called Bell measurement [3]) on her own qubit and the qubit of the EPR pair that she holds. The Bell measurement random outcome can be unambiguously identified by two classical bits (cbits) which are by no means related to content of the information previously contained in Alice's original qubit. Conditioned on the two cbits that Alice announces, Bob is able to "open" the desired information from the qubit of the EPR pair that he holds. Thus, the information is securely and faithfully "flown" from a qubit at Alice's to another qubit at Bob's, without any real matter portation. Such a quantum intriguing protocol is now widely known under a quite fictitious name 'quantum teleportation' first invented in 1993 [4]. For global tasks involving many participants, such as controlled teleportation [5, 6], quantum secret sharing [7], joint remote state preparation [8,9] and others, multiqubit maximally entangled states are needed. In some tasks one authorized party may hold more than one qubit of the shared multiqubit entanglement.

The trouble is that the authorized parties are usually located at large (even possibly spacelike) distances and, due to unavoidable interaction with surrounding environments, after an actual entanglement distribution process the entanglement degree becomes degraded (i.e., the initial maximally entangled state turns out to be a nonmaximally entangled one) and the entanglement degradation rate grows exponentially with distance that badly influences success and quality of the intended task. Generally, when the quantum channel suffers from a phase damping noise the entangled state takes an infinite time to become completely separable, a phenomenon called entanglement asymptotic death (EAD), but when the noise is of amplitude damping type the entanglement may vanish suddenly, a phenomenon called entanglement sudden death (ESD) [10]. In cases both phase damping and amplitude damping are simultaneously active then the transition of entanglement to complete separability may be either asymptotic or sudden depending on the entangled state under consideration [11]. To overcome such a negative affect of the environment one can first process the entanglement sharing repeatedly so that many copies of degraded entangled states are shared and then implement a LOCC-assisted method to transform these many degraded nonmaximally entangled states into a desired maximally entangled one. This procedure is referred to as entanglement distillation, which was originally devised in [12] for pure states and in [13] for mixed states. Despite the usefulness of entanglement distillation procedure, its overall cost is high because many rounds of entanglement shares and many applications of local 
operations/measurements as well as a lot of classical communication are consumed. Also, the cost greatly increases with the number of qubits that comprise the interested entangled state. Therefore, economizing nonlocal resource (i.e., the number of shared entangled qubits) is extremely crucial, especially when dealing with multiqubit entangled resource.

In this paper, we revisit a recently published protocol on 'Two-Way Remote Preparations of Inequivalent Quantum States Under a Common Control' [14] in which Alice can prepare for her remote Bob a three-qubit Greenberger-Horne-Zeilinger-type (GHZ-type) state [15] of the form

$$
|\chi\rangle=\alpha_{0}|000\rangle+\alpha_{1}|111\rangle
$$

while, at the same time, Bob can prepare for Alice an inequivalent state of the form of a four-qubit W-type state [16]

$$
|\tau\rangle=\beta_{0}|0001\rangle+\beta_{1}|0010\rangle+\beta_{2}|0100\rangle+\beta_{3}|1000\rangle
$$

in such a way that the above-mentioned remote state preparations in both directions (i.e., from Alice to Bob as well as from Bob to Alice) can only be done upon decision of a common controller Charlie. In the above equations (1) and (2) $|000\rangle \equiv|0\rangle \otimes|0\rangle \otimes|0\rangle,|0001\rangle \equiv|0\rangle \otimes|0\rangle \otimes|0\rangle \otimes|1\rangle$ and so on. For simplicity, the coefficients $\left\{\alpha_{i} ; i=0,1\right\}$ and $\left\{\beta_{j} ; j=0,1,2,3\right\}$ in Eqs. (1) and (2) are assumed real and satisfy the normalization constraints $\sum_{i=0}^{1} \alpha_{i}^{2}=\sum_{j=0}^{3} \beta_{j}^{2}=1$. Furthermore, the coefficients $\alpha_{0}$ and $\alpha_{1}$ are known only to Alice, while Bob is the only one who knows the coefficients $\beta_{0}, \beta_{1}, \beta_{2}$ and $\beta_{3}$. The shared nonlocal resource in [14] was a maximally entangled state that consists of eleven qubits which leads to an expensive cost for the process of distributing the qubits among Alice, Bob and Charlie, as explained before. Here we have found out that the same tasks as formulated in [14] can be executed equally well by using a nonlocal resource which is made only of seven qubits, a much more economical protocol as compared to the original one. The details of how to use the seven-qubit nonlocal resource for the required remote state preparations will be presented in the section II. Section III proposes a scheme to generate the nonlocal resource used in Section II. The final section, Section IV, will be reserved for discussion and conclusion.

\section{SEVEN-QUBIT NONLOCAL RESOURCE: HOW DOES IT WORK?}

As the working quantum channel, instead of the eleven-qubit entangled state given in Eq. (3) of Ref. [14]), here we consider a much simpler one which is made only of seven qubits of the form

$$
\begin{aligned}
|q\rangle= & \frac{1}{2 \sqrt{2}}(|0000000\rangle+|0010011\rangle \\
& +|0110110\rangle+|0100101\rangle \\
& +|1011010\rangle+|1001001\rangle \\
& +|1101100\rangle+|1111111\rangle)_{a_{1} a_{2} a_{3} b_{1} b_{2} b_{3} c}
\end{aligned}
$$

of which qubits $a_{1}, a_{2}, a_{3}$ are possessed by Alice, qubits $b_{1}, b_{2}, b_{3}$ by Bob and qubit $c$ by Charlie. Let us address on the issue of how to generate the above quantum channel state $|q\rangle$ later, but rush right now to the issue of how it works. 
With the qubits $a_{1}, a_{2}, a_{3}$ at hand Alice manages to manipulate them locally as she wants. Likewise, Bob is able to perform any local operations including unitary logic gates and measurements on his qubits $b_{1}, b_{2}, b_{3}$ and so does Charlie with her qubit $c$. For the purpose of two-way remote state preparations under a common control as described in the section Introduction, first Alice and Bob do their operations and then Charlie does hers. Namely, Alice measures qubit $a_{1}$ in the basis $\left\{\left|u_{0}\right\rangle_{a_{1}},\left|u_{1}\right\rangle_{a_{1}}\right\}$ with which the computational basis states $\left\{|0\rangle_{a_{1}},|1\rangle_{a_{1}}\right\}$ are unitarily related as

$$
\left(\begin{array}{l}
|0\rangle_{a_{1}} \\
|1\rangle_{a_{1}}
\end{array}\right)=\left(\begin{array}{cc}
\alpha_{0} & -\alpha_{1} \\
\alpha_{1} & \alpha_{0}
\end{array}\right)\left(\begin{array}{l}
\left|u_{0}\right\rangle_{a_{1}} \\
\left|u_{1}\right\rangle_{a_{1}}
\end{array}\right)
$$

Independently, Bob carries out a joint measurement on qubits $b_{2}, b_{3}$ in the basis $\left\{\left|v_{00}\right\rangle_{b_{2} b_{3}},\left|v_{01}\right\rangle_{b_{2} b_{3}}\right.$, $\left.\left|v_{10}\right\rangle_{b_{2} b_{3}},\left|v_{11}\right\rangle_{b_{2} b_{3}}\right\}$ with which the computational basis states $\left\{|00\rangle_{b_{2} b_{3}},|01\rangle_{b_{2} b_{3}},|10\rangle_{b_{2} b_{3}},|11\rangle_{b_{2} b_{3}}\right\}$ are unitarily related as

$$
\left(\begin{array}{l}
|00\rangle_{b_{2} b_{3}} \\
|01\rangle_{b_{2} b_{3}} \\
|10\rangle_{b_{2} b_{3}} \\
|11\rangle_{b_{2} b_{3}}
\end{array}\right)=\left(\begin{array}{cccc}
\beta_{0} & \beta_{1} & -\beta_{2} & \beta_{3} \\
\beta_{1} & -\beta_{0} & \beta_{3} & \beta_{2} \\
\beta_{2} & \beta_{3} & \beta_{0} & -\beta_{1} \\
\beta_{3} & -\beta_{2} & -\beta_{1} & -\beta_{0}
\end{array}\right)\left(\begin{array}{l}
\left|v_{00}\right\rangle_{b_{2} b_{3}} \\
\left|v_{01}\right\rangle_{b_{2} b_{3}} \\
\left|v_{10}\right\rangle_{b_{2} b_{3}} \\
\left|v_{11}\right\rangle_{b_{2} b_{3}}
\end{array}\right) .
$$

Note that Alice and Bob can act independently from each other, i.e., Alice may do her measurement first or they even do their measurements simultaneously. In other words, the order of their measurements does not matter.

For Alice's measurement there are two probabilistic outcomes which can be specified by one cbit $l \in\{0,1\}: l=0$ specifies the outcome $\left|u_{0}\right\rangle_{a_{1}}$ but $l=1$ implies the outcome $\left|u_{1}\right\rangle_{a_{1}}$. Concerning Bob's measurement there are four possible outcomes which can be specified by two cbits $m n \in\{00,01,10,11\}: m n=00,01,10$ or 11, if $\left|v_{00}\right\rangle_{b_{2} b_{3}},\left|v_{01}\right\rangle_{b_{2} b_{3}},\left|v_{10}\right\rangle_{b_{2} b_{3}}$ or $\left|v_{11}\right\rangle_{b_{2} b_{3}}$ are found, respectively. The state $|q\rangle$ of the working quantum channel in Eq.(3) can be rewritten in terms of $\left\{\left|u_{l}\right\rangle_{a_{1}}\right\}$ and $\left\{\left|v_{m n}\right\rangle_{b_{2} b_{3}}\right\}$ as

$$
|q\rangle=\frac{1}{2 \sqrt{2}} \sum_{l, m, n=0}^{1}\left|u_{l}\right\rangle_{a_{1}}\left|v_{m n}\right\rangle_{b_{2} b_{3}}\left|w_{m n l}\right\rangle_{a_{2} a_{3} b_{1} c}
$$

with $\left|w_{m n l}\right\rangle_{a_{2} a_{3} b_{1} c}$ to be determined shortly.

In principle, if the measurement outcomes $m n$ and $l$ are disclosed publicly, then Alice could transform her qubits $a_{2}, a_{3}$ to the desired state $|t\rangle_{a_{2} a_{3}}$ and, at the same time, Bob could transform his qubit $b_{1}$ to the desired state $|x\rangle_{b_{1}}$. However, at this moment they could not, because the qubits $a_{2}, a_{3}$ and $b_{1}$ remain entangled with Charlie's qubit $c$, indicating a decisive role of the controller Charlie. If Charlie, by some reasons, does not want the state preparations to be completed, she simply does nothing. Otherwise, she measures her qubit $c$ in the basis $\left\{|\widetilde{0}\rangle_{c},|\widetilde{1}\rangle_{c}\right\}$, with

$$
|\widetilde{0}\rangle_{c} \equiv \frac{1}{\sqrt{2}}(|0\rangle+|1\rangle)_{c}
$$

and

$$
|\tilde{1}\rangle_{c} \equiv \frac{1}{\sqrt{2}}(|0\rangle-|1\rangle)_{c} .
$$


As a rule, Charlie's outcome is either $|\widetilde{0}\rangle_{c}$, which is assigned with a cbit $k=0$, or $|\tilde{1}\rangle_{c}$, which is assigned with a cbit $k=1$. In terms of $\left\{|\widetilde{k}\rangle_{c}\right\}$ the expression of states $\left|w_{m n l}\right\rangle_{a_{2} a_{3} b_{1} c}$ in Eq. (6) can be derived explicitly as

$$
\left|w_{m n l}\right\rangle_{a_{2} a_{3} b_{1} c}=\frac{1}{\sqrt{2}} \sum_{k=0}^{1}\left|t_{m n k}\right\rangle_{a_{2} a_{3}}\left|x_{l k}\right\rangle_{b_{1}}|\widetilde{k}\rangle_{c}
$$

where

and

$$
\begin{gathered}
\left|t_{000}\right\rangle_{a_{2} a_{3}}=\left(\beta_{0}|00\rangle+\beta_{1}|01\rangle+\beta_{2}|10\rangle+\beta_{3}|11\rangle\right)_{a_{2} a_{3}}, \\
\left|t_{001}\right\rangle_{a_{2} a_{3}}=\left(\beta_{0}|00\rangle-\beta_{1}|01\rangle-\beta_{2}|10\rangle+\beta_{3}|11\rangle\right)_{a_{2} a_{3}}, \\
\left|t_{010}\right\rangle_{a_{2} a_{3}}=\left(\beta_{1}|00\rangle-\beta_{0}|01\rangle+\beta_{3}|10\rangle-\beta_{2}|11\rangle\right)_{a_{2} a_{3}}, \\
\left|t_{011}\right\rangle_{a_{2} a_{3}}=\left(\beta_{1}|00\rangle+\beta_{0}|01\rangle-\beta_{3}|10\rangle-\beta_{2}|11\rangle\right)_{a_{2} a_{3}}, \\
\left|t_{100}\right\rangle_{a_{2} a_{3}}=\left(-\beta_{2}|00\rangle+\beta_{3}|01\rangle+\beta_{0}|10\rangle-\beta_{1}|11\rangle\right)_{a_{2} a_{3}}, \\
\left|t_{101}\right\rangle_{a_{2} a_{3}}=\left(\beta_{2}|00\rangle+\beta_{3}|01\rangle+\beta_{0}|10\rangle+\beta_{1}|11\rangle\right)_{a_{2} a_{3}}, \\
\left|t_{110}\right\rangle_{a_{2} a_{3}}=\left(\beta_{3}|00\rangle+\beta_{2}|01\rangle-\beta_{1}|10\rangle-\beta_{0}|11\rangle\right)_{a_{2} a_{3}}, \\
\left|t_{111}\right\rangle_{a_{2} a_{3}}=\left(\beta_{3}|00\rangle-\beta_{2}|01\rangle+\beta_{1}|10\rangle-\beta_{0}|11\rangle\right)_{a_{2} a_{3}}
\end{gathered}
$$

$$
\begin{aligned}
& \left|x_{00}\right\rangle_{b_{1}}=\left(\alpha_{0}|0\rangle+\alpha_{1}|1\rangle\right)_{b_{1}}, \\
& \left|x_{01}\right\rangle_{b_{1}}=\left(\alpha_{0}|0\rangle-\alpha_{1}|1\rangle\right)_{b_{1}}, \\
& \left|x_{10}\right\rangle_{b_{1}}=\left(\alpha_{1}|0\rangle-\alpha_{0}|1\rangle\right)_{b_{1}}, \\
& \left|x_{11}\right\rangle_{b_{1}}=\left(\alpha_{1}|0\rangle+\alpha_{0}|1\rangle\right)_{b_{1}} .
\end{aligned}
$$

Substitution of (9) into (6) yields

$$
|q\rangle=\frac{1}{4} \sum_{l, m, n, k=0}^{1}\left|u_{l}\right\rangle_{a_{1}}\left|t_{m n k}\right\rangle_{a_{2} a_{3}}\left|x_{l k}\right\rangle_{b_{1}}\left|v_{m n}\right\rangle_{b_{2} b_{3}}|\widetilde{k}\rangle_{c} .
$$

As followed from Eq. (22), if outcomes of Alice's, Bob's and Charlie's measurements are respectively $l, m n$ and $k$, then the state of Alice's qubits $a_{2}, a_{3}$ and that of Bob's qubit $b_{1}$ are disentangled from Charlie's qubit $c$. More than that, qubits $a_{2}, a_{3}$ and qubit $b_{1}$ are also disentangled: qubits $a_{2}, a_{3}$ are projected onto $\left|t_{m n k}\right\rangle_{a_{2} a_{3}}$, while qubits $b_{1}$ onto $\left|x_{l k}\right\rangle_{b_{1}}$, whose explicit forms are given in Eqs. (10) - (21). That is, the state of qubits $a_{2}, a_{3}$ depends on Bob's measurement outcomes $m n$, the state of qubit $b_{1}$ depends on Alice's measurement outcome $l$, and both the states depend on Charlie's measurement outcome $k$. Conditioned on all the obtained measurement outcomes, which are announced through a public, insecure yet reliable, classical channel, Alice is able to transform $\left|t_{m n k}\right\rangle_{a_{2} a_{3}}$ to

$$
|t\rangle_{a_{2} a_{3}}=\left(\beta_{0}|00\rangle+\beta_{1}|01\rangle+\beta_{2}|10\rangle+\beta_{3}|11\rangle\right)_{a_{2} a_{3}}
$$

by applying on $\left|t_{m n k}\right\rangle_{a_{2} a_{3}}$ the unitary operator

$$
A_{m n k}=X_{a_{2}}^{m} Z_{a_{2}}^{m \oplus k} \otimes X_{a_{3}}^{n} Z_{a_{3}}^{m \oplus n \oplus k},
$$

while, simultaneously, Bob is able to transform $\left|x_{l k}\right\rangle_{b_{1}}$ to

$$
|x\rangle_{b_{1}}=\left(\alpha_{0}|0\rangle+\alpha_{1}|1\rangle\right)_{b_{1}}
$$


by applying on $\left|x_{l k}\right\rangle_{b_{1}}$ the unitary operator

$$
B_{l k}=X_{b_{1}}^{l} Z_{b_{1}}^{l \oplus k}
$$

In Eqs. (24) and (26)

$$
X=\left(\begin{array}{ll}
0 & 1 \\
1 & 0
\end{array}\right)
$$

denotes the bit-flip operator and

$$
Z=\left(\begin{array}{cc}
1 & 0 \\
0 & -1
\end{array}\right)
$$

the phase-flip operator, with $\oplus$ being an addition modulus 2 and $\otimes$ the tensor product. For illustration, the outcomes $\{l, m n, k\}=\{0,00,0\}$ lead to $A_{000}=I_{a_{2}} \otimes I_{a_{3}}$ and $B_{00}=I_{b_{1}}$, with $I_{a_{2}}, I_{a_{3}}, I_{b_{1}}$ the identity operators, implying doing nothing. Another set of outcomes $\{l, m n, k\}=\{0,00,1\}$ leads to other operators $A_{001}=Z_{a_{2}} \otimes Z_{a_{3}}$ and $B_{01}=Z_{b_{1}}$, implying application of a phase-flip operator on each of the qubits $a_{2}, a_{3}$ and $b_{1}$, etc.

After having the states $|t\rangle_{a_{2} a_{3}}$ and $|x\rangle_{b_{1}}$ of Eqs. (23) and (25), Alice and Bob need employing some additional local operators which turn out to be Controlled-NOT gate (CNOT):

$$
\mathrm{CNOT}_{a b}|p\rangle_{a}|q\rangle_{b}=|p\rangle_{a}|q \oplus p\rangle_{b}
$$

i.e., if the cbit of the first qubit $a$ is 1 , then the cbit of the second qubit $b$ is flipped, otherwise nothing happens and Controlled-Controlled-NOT gate (CCNOT):

$$
\mathrm{CCNOT}_{a b c}|p\rangle_{a}|q\rangle_{b}|r\rangle_{c}=|p\rangle_{a}|q\rangle_{b}|r \oplus p q\rangle_{c},
$$

i.e., if both the cbits of the two first qubits $a, b$ are 1, then the cbit of the third qubit $c$ is flipped, otherwise all the cbits remain unchanged. Although CNOT is quite commonplace, CCNOT is not. In the literature the CCNOT is also referred to as Toffoli gate which was invented in 1980 by Tommaso Toffoli [17]. In fact, CCNOT is a three-qubit logic gate but it can be realized by at least five two-qubit logic gates combined with single-qubit logic gates [18]. Returning to our protocol, Bob prepares two ancillary qubits $b_{1}^{\prime}$ and $b_{1}^{\prime \prime}$ in product state $|0\rangle_{b_{1}^{\prime}}|0\rangle_{b_{1}^{\prime \prime}}$, then applies two CNOTs, $C N O T_{b_{1} b_{1}^{\prime}}$ and $C N O T_{b_{1} b_{1}^{\prime \prime}}$, on his three qubits $b_{1}, b_{1}^{\prime}$ and $b_{1}^{\prime \prime}$. It is easy to check that

$$
C N O T_{b_{1} b_{1}^{\prime \prime}} C N O T_{b_{1} b_{1}^{\prime}}|x\rangle_{b_{1}}|0\rangle_{b_{1}^{\prime}}|0\rangle_{b_{1}^{\prime \prime}}=|\chi\rangle_{b_{1} b_{1}^{\prime} b_{1}^{\prime \prime}}
$$

which is exactly the three-qubit GHZ-type state (1) that Alice aims to prepare for Bob. Concerning Alice, she also prepares two ancillary qubits $a_{2}^{\prime}$ and $a_{3}^{\prime}$ in product state $|0\rangle_{a_{2}^{\prime}}|1\rangle_{a_{3}^{\prime}}$, then applies on her four qubits $a_{2}, a_{3}, a_{2}^{\prime}$ and $a_{3}^{\prime}$ an operator $U_{a_{2} a_{3} a_{2}^{\prime} a_{3}^{\prime}}$ which is composed from several CNOTs and one CCNOT as

$$
U_{a_{2} a_{3} a_{2}^{\prime} a_{3}^{\prime}}=\mathrm{CNOT}_{a_{2}^{\prime} a_{2}} \mathrm{CNOT}_{a_{2}^{\prime} a_{3}} \mathrm{CNOT}_{a_{2}^{\prime} a_{3}^{\prime}} \mathrm{CCNOT}_{a_{2} a_{3} a_{2}^{\prime}} \mathrm{CNOT}_{a_{3} a_{3}^{\prime}} \mathrm{CNOT}_{a_{2} a_{3}^{\prime}} .
$$

It is quite nontrivial but not difficult to verify that

$$
U_{a_{2} a_{3} a_{2}^{\prime} a_{3}^{\prime}}|t\rangle_{a_{2} a_{3}}|0\rangle_{a_{2}^{\prime}}|1\rangle_{a_{3}^{\prime}}=|\tau\rangle_{a_{2}^{\prime} a_{2} a_{3} a_{3}^{\prime}}
$$

which is exactly the four-qubit W-type state (2) that Bob aims to prepare for Alice. Derivation of Eq. (33) is done in the Appendix. 


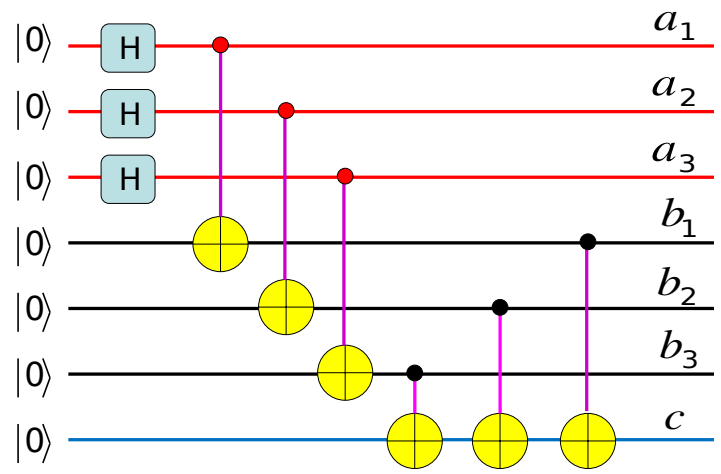

Fig. 1. Quantum circuit generating the state $|q\rangle$ in Eq. (3) of the seven-qubit nonlocal resource. A qubit is represented by a horizontal line. $H$ stands for a single-qubit Hadamard gate in Eq. (35). A controlled-NOT gate (CNOT) in Eq.(29) is represented by a vertical straight segment connecting a filled circle $\bullet$ belonging to the control qubit and a NOT gate $\oplus$ belonging to the target qubit. Time goes from left to right and all the seven qubits are prepared in state $|0\rangle$ initially.

\section{SEVEN-QUBIT NONLOCAL RESOURCE: HOW IS IT GENERATED?}

The protocol for the two-way remote preparations of inequivalent quantum states under a common control presented in the previous section would be meaningless if the seven-qubit entangled state $|q\rangle$ defined by Eq. (3) does not really exist. So, in this section we propose a possible scheme to generate that state. Suppose that David, a technical employee of a company specializing in the production of various quantum states, is in charge for the generation of the state $|q\rangle$. David can go along the following steps.

First, he initializes the generation process by taking seven qubits $a_{1}, a_{2}, a_{3}, b_{1}, b_{2}, b_{3}$ and $c$, all in the states $|0\rangle$, i.e., the initial state is

$$
\left|q_{0}\right\rangle=|0\rangle_{a_{1}}|0\rangle_{a_{1}}|0\rangle_{a_{3}}|0\rangle_{b_{1}}|0\rangle_{b_{2}}|0\rangle_{b_{3}}|0\rangle_{c} .
$$

Second, three Hadamard gates,

$$
H=\frac{1}{\sqrt{2}}\left(\begin{array}{cc}
1 & 1 \\
1 & -1
\end{array}\right)
$$

are applied on qubits $a_{1}, a_{2}$ and $a_{3}$, bringing $\left|q_{0}\right\rangle$ to

$$
\begin{aligned}
\left|q_{1}\right\rangle & =H_{a_{1}} H_{a_{2}} H_{a_{3}}\left|q_{0}\right\rangle \\
& =|\widetilde{0}\rangle_{a_{1}}|\widetilde{0}\rangle_{a_{1}}|\widetilde{0}\rangle_{a_{3}}|0\rangle_{b_{1}}|0\rangle_{b_{2}}|0\rangle_{b_{3}}|0\rangle_{c}
\end{aligned}
$$

with $|\widetilde{0}\rangle$ defined by Eq. (7). 
Third, three CNOTs, $C N O T_{a_{1} b_{1}}, C N O T_{a_{2} b_{2}}$ and $C N O T_{a_{3} b_{3}}$, are applied on $\left|q_{1}\right\rangle$ bringing it to $\left|q_{2}\right\rangle$ of the following form

$$
\begin{aligned}
\left|q_{2}\right\rangle & =\operatorname{CNOT}_{a_{1} b_{1}} \operatorname{CNOT}_{a_{2} b_{2}} \operatorname{CNOT}_{a_{3} b_{3}}\left|q_{1}\right\rangle \\
& =|E P R\rangle_{a_{1} b_{1}}|E P R\rangle_{a_{2} b_{2}}|E P R\rangle_{a_{3} b_{3}}|0\rangle_{c}
\end{aligned}
$$

with

$$
|E P R\rangle_{a b}=\frac{1}{\sqrt{2}}\left(|0\rangle_{a}|0\rangle_{b}+|1\rangle_{a}|1\rangle_{b}\right)
$$

being one of the four EPR states [1].

Finally, three more CNOTs, $C N O T_{b_{1} c}, C N O T_{b_{2} c}$ and $C N O T_{b_{3} c}$, are applied on $\left|q_{2}\right\rangle$ to have

$$
\left|q_{3}\right\rangle=\mathrm{CNOT}_{b_{1} c} \mathrm{CNOT}_{b_{2} c} \mathrm{CNOT}_{b_{3} c}\left|q_{2}\right\rangle \text {. }
$$

By putting $\left|q_{2}\right\rangle$ of Eq. (37) into the right-hand-side of Eq. (39) and calculating $\left|q_{3}\right\rangle$ we will see that $\left|q_{3}\right\rangle$ is nothing else but the desired seven-qubit entangled state $|q\rangle$. The quantum circuit for generation of the state $|q\rangle$ is shown in Fig.1.

Having generated such a seven-qubit entangled state David distributes qubits $a_{1}, a_{2}, a_{3}$ to Alice, qubits $b_{1}, b_{2}, b_{3}$ to Bob and qubit $c$ to Charlie across noisy environments. The parties should employ an entanglement distillation process until successfully sharing a pure state $|q\rangle$ of the form given in Eq. (3). Only after that Alice, Bob and Charlie start the two-way remote state preparations detailed in the previous section.

\section{DISCUSSION AND CONCLUSION}

In the quantum world 'nothing exists until it is measured' [19]. Alice, Bob and Charlie never know in advance their measurement outcomes which occur non-deterministically. In this protocol, an outcome of Alice's measurement associated with a specific value of cbit $l(l=0$ or 1) happens absolutely randomly, i.e, with a probability of $1 / 2$, an outcome of Bob's measurement associated with specific values of two cbits $m n(m n=00,01,10$ or 11) also happens randomly with an equal probability of $1 / 4$, and so is an outcome of Charlie's measurement which is associated with a specific value of cbit $k(k=0$ or 1$)$ happening with a probability of $1 / 2$. Therefore, a particular set of measurement outcomes associated with specific values of cbits $l, m n$ and $k$ occurs with a probability of $1 / 16$. Despite of this probabilistic feature, our protocol described in Section II is deterministic, i.e., it always succeeds, because for whatever set of possible values of $l, m n$ and $k$ the necessary transformation operators (24) and (26) exist in terms of well-defined single-qubit logic gates.

Using the same nonlocal resource in form of the seven-qubit state (3) Alice and Bob can exchange inequivalent states other than those given in Eqs. (1) and (2). Namely, instead of (1) Alice can also prepare for Bob an $N$-qubit GHZ-type state of the form $|\Xi\rangle=\alpha_{0}|00 \ldots 0\rangle+$ $\alpha_{1}|11 \ldots 1\rangle_{b_{1} B_{2} \ldots B_{N}}$. For that purpose Bob takes $N-1$ ancillas in state $|0\rangle_{B_{2}}|0\rangle_{B_{3}} \ldots|0\rangle_{B_{N}}$ and applies $N-1$ CNOTs, $C N O T_{b_{1} B_{2}}, C N O T_{b_{1} B_{3}}, \ldots$ and $C N O T_{b_{1} B_{N}}$ on $|x\rangle_{b_{1}}|0\rangle_{B_{2}}|0\rangle_{B_{3}} \ldots|0\rangle_{B_{N}}$ to readily obtain $|\Xi\rangle$. From his side, Bob can also prepare for Alice a cluster state [20]. For that purpose Alice takes an additional state $|0\rangle_{a_{2}^{\prime}}|0\rangle_{a_{3}^{\prime}}$ of two ancillary qubits $a_{2}^{\prime}$ and $a_{3}^{\prime}$ and applies $C N O T_{a_{2} a_{2}^{\prime}} C N O T_{a_{3} a_{3}^{\prime}}$ on $|t\rangle_{a_{2} a_{3}}|0\rangle_{a_{2}^{\prime}}|0\rangle_{a_{3}^{\prime}}$ to obtain $|T\rangle=\left(\beta_{0}|0000\rangle+\beta_{1}|0101\rangle+\beta_{2}|1010\rangle+\beta_{3}|1111\rangle\right)_{a_{2} a_{3} a_{2}^{\prime} a_{3}^{\prime}}$ which is a type of four-qubit cluster state. 
In conclusion, we reconsider the problem which was recently addressed on in Ref. [14]. The merit of this reconsideration is that the nonlocal resource employed in [14] is greatly economized from using eleven qubits to only seven ones. Furthermore, using the seven-qubit entanglement (3) allows two-way preparations of inequivalent states other than the states (1) and (2) touched upon in [14]. Actually, the total number of used qubits is the same in [14] and here. However, in [14] all the eleven qubits contribute to the nonlocal resource in terms of an eleven-qubit entangled state, while here the nonlocal resource is built only by seven qubits and the remaining four qubits just serve as auxiliary local resources. As local qubits do not need to be shared among the authorized parties, the noise due to environment across which distribution of nonlocal qubits takes place, has no influence on them. This means that the lesser the number of nonlocal qubits the cheaper the overall cost to perform a given task. Therefore, any possible economization of nonlocal resource is worth in practice. The idea of exploiting local resource to economize nonlocal resource was deployed previously in different contexts [21,22].

\section{ACKNOWLEDGMENTS}

This work is supported by National Foundation for Science and Technology Development (NAFOSTED) under Project No. 103.01-2019.313.

\section{REFERENCES}

[1] A. Einstein, B. Podolsky and N. Rosen, Phys. Rev. 47 (1935) 777.

[2] E. Schrödinger, Naturwissenschaften 23 (1935) 807.

[3] J. S. Bell, Physics 1 (1964) 195.

[4] C. H. Bennett, G. Brassard, C. Crépeau, R. Jozsa, A. Peres, and W. K. Wootters, Phys. Rev. Lett. 70 (1993) 1895.

[5] A. Karlsson and M. Bourennane, Phys. Rev. A 58 (1998) 4394.

[6] N. B. An, Phys. Rev. A 68 (2003) 022321.

[7] M. Hillery, V. Bužek, and A. Berthiaume, Phys. Rev. A 59 (1998) 1829.

[8] N. B. An and J. Kim, J. Phys. B: At. Mol. Opt. Phys. 41 (2008) 095501.

[9] N. B. An, Optics Communications 283 (2010) 4113.

[10] T. Yu and J. H. Eberly, Phys. Rev. Lett. 97 (2006) 140403.

[11] N. B. An and J. Kim, Phys. Rev. A 79 (2009) 022303.

[12] C. H. Bennett, H. Bernstein, S. Popescu, and B. Schumacher, Phys. Rev. A 53 (1996) 2046.

[13] C. H. Bennett, G. Brassard, S. Popescu, B. Schumacher, J. A. Smolin, and W. K. Wooters, Phys. Rev. Lett. 76 (1996) 722.

[14] N. B. An, B. S. Choudhury and S. Samanta, Int. J. Theor. Phys. 60 (2021) 47.

[15] D. M. Greenberger, M. A. Horne and A. Zeilinger, in: Bells Theorem, Quantum Theory, and Conception of the Universe, M. Kafatos (ed.), Kluwer, Dordrecht, 1989, p. 69.

[16] W. Dür, G. Vidal and J. I. Cirac, Phys. Rev. A 62 (2000) 062314.

[17] T. Toffoli, in: Reversible computing, J. W. de Bakker and J. van Leeuwen (ed.), 1980.

[18] N. Yu, R. Duan and M. Ying, Phys. Rev. A 88 (2013) 010304R.

[19] A quote by Niels Bohr (see, e.g., https://quotefancy.com/quote/1009160/Niels-Bohr-Nothing-exists-until-it-ismeasured)

[20] H. J. Briegel and R. Raussendorf, Phys. Rev. Lett. 86 (2001) 910.

[21] N. B. An, Phys. Lett. A 341 (2005) 9.

[22] N. B. An, C. T. Bich and N. V. Don, J. Phys. B: At. Mol. Opt. Phys. 44 (2011) 135506. 


\section{APPENDIX}

This Appendix verifies the equality (33). The verification goes as follows.

$U_{a_{2} a_{3} a_{2}^{\prime} a_{3}^{\prime}}|t\rangle_{a_{2} a_{3}}|0\rangle_{a_{2}^{\prime}}|1\rangle_{a_{3}^{\prime}}$

$=$ CNOT $_{a_{2}^{\prime} a_{2}}{ } N^{\prime} O T_{a_{2}^{\prime} a_{3}} C N O T_{a_{2}^{\prime} a_{3}^{\prime}} C^{\prime} C N O T_{a_{2} a_{3} a_{2}^{\prime}}$ CNOT $_{a_{3} a_{3}^{\prime}}$ CNOT $_{a_{2} a_{3}^{\prime}}$

$\left(\beta_{0}|00\rangle+\beta_{1}|01\rangle+\beta_{2}|10\rangle+\beta_{3}|11\rangle\right)_{a_{2} a_{3}}|0\rangle_{a_{2}^{\prime}}|1\rangle_{a_{3}^{\prime}}$

$=\mathrm{CNOT}_{a_{2}^{\prime} a_{2}} \mathrm{CNOT}_{a_{2}^{\prime} a_{3}} \mathrm{CNOT}_{a_{2}^{\prime} a_{3}^{\prime}} \mathrm{CCNOT}_{a_{2} a_{3} a_{2}^{2}} \mathrm{CNOT}_{a_{3} a_{3}^{\prime}} \mathrm{CNOT}_{a_{2} a_{3}^{\prime}}$

$\left(\beta_{0}|0001\rangle+\beta_{1}|0101\rangle+\beta_{2}|1001\rangle+\beta_{3}|1101\rangle\right)_{a_{2} a_{3} a_{2}^{\prime} a_{3}^{\prime}}$

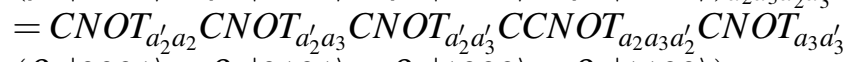

$\left(\beta_{0}|0001\rangle+\beta_{1}|0101\rangle+\beta_{2}|1000\rangle+\beta_{3}|1100\rangle\right)_{a_{2} a_{3} a_{2}^{\prime} a_{3}^{\prime}}$

$=\mathrm{CNOT}_{a_{2}^{\prime} a_{2}} \mathrm{CNOT}_{a_{2}^{\prime} a_{3}} C N O T_{a_{2}^{\prime} a_{3}^{\prime}} C \mathrm{CNOT} \mathrm{a}_{a_{2} a_{3} a_{2}^{\prime}}$

$\left(\beta_{0}|0001\rangle+\beta_{1}|0100\rangle+\beta_{2}|1000\rangle+\beta_{3}|1101\rangle\right)_{a_{2} a_{3} a_{2}^{\prime} a_{3}^{\prime}}$

$=C N O T_{a_{2}^{\prime} a_{2}} C N O T_{a_{2}^{\prime} a_{3}} C N O T_{a_{2}^{\prime} a_{3}^{\prime}}\left(\beta_{0}|0001\rangle+\beta_{1}|0100\rangle+\beta_{2}|1000\rangle+\beta_{3}|1111\rangle\right)_{a_{2} a_{3} a_{2}^{\prime} a_{3}^{\prime}}$

$=C N O T_{a_{2}^{\prime} a_{2}} C N O T_{a_{2}^{\prime} a_{3}}\left(\beta_{0}|0001\rangle+\beta_{1}|0100\rangle+\beta_{2}|1000\rangle+\beta_{3}|1110\rangle\right)_{a_{2} a_{3} a_{2}^{\prime} a_{3}^{\prime}}$

$=\operatorname{CNOT}_{a_{2}^{\prime} a_{2}}\left(\beta_{0}|0001\rangle+\beta_{1}|0100\rangle+\beta_{2}|1000\rangle+\beta_{3}|1010\rangle\right)_{a_{2} a_{3} a_{2}^{\prime} a_{3}^{\prime}}$

$=\left(\beta_{0}|0001\rangle+\beta_{1}|0100\rangle+\beta_{2}|1000\rangle+\beta_{3}|0010\rangle\right)_{a_{2} a_{3} a_{2}^{\prime} a_{3}^{\prime}}$.

Changing the label order $a_{2} a_{3} a_{2}^{\prime} a_{3}^{\prime} \rightarrow a_{2} a_{2}^{\prime} a_{3} a_{3}^{\prime}$ and then $a_{2} a_{2}^{\prime} a_{3} a_{3}^{\prime} \rightarrow a_{2}^{\prime} a_{2} a_{3} a_{3}^{\prime}$ yields

$|\tau\rangle_{a_{2}^{\prime} a_{2} a_{3} a_{3}^{\prime}}$

$U_{a_{2} a_{3} a_{2}^{\prime} a_{3}^{\prime}}|t\rangle_{a_{2} a_{3}}|0\rangle_{a_{2}^{\prime}}|1\rangle_{a_{3}^{\prime}}=\left(\beta_{0}|0001\rangle+\beta_{1}|0010\rangle+\beta_{2}|0100\rangle+\beta_{3}|1000\rangle\right)_{a_{2}^{\prime} a_{2} a_{3} a_{3}^{\prime}}$ which is 